\title{
AMENABLE TRANSFORMATION SEMIGROUPS
}

\author{
CARROLL WILDE and TOKE JAYACHANDRAN ${ }^{1}$
}

(Received 19 June 1969)

Communicated by G. B. Preston

\section{Introduction}

For any set $X$ denote by $m(X)$ the Banach space of all bounded real-valued functions on $X$, equipped with the supremum norm, and denote by $\subseteq(X)$ the semigroup (under functional composition) of all transformations of $X$, i.e. mappings with domain $X$ and range contained in $X$. A pair $(X, S)$, where $S$ is a subsemigroup of $\subseteq(X)$, will be called a transformation semigroup. Important examples are obtained by letting $X$ be the underlying set in an abstract semigroup and considering the pairs $\left(X, S_{1}\right)$ and $\left(X, S_{2}\right)$, where $S_{1}\left[S_{2}\right]$ denotes the set of left [right] multiplication mappings of $X$. We shall call transformation semigroups in these classes of examples $l$ - $[r-]$ semigroups.

A mean on $m(X)$ is a positive normalized continuous linear functional on $m(X)$, i.e. an element $\mu$ in $m(X)^{*}$ such that $\mu(f) \geqq 0$ if $f(x) \geqq 0$ for all $x$ in $X$, and such that $\mu(1)=1$, where 1 is the function $1(x)=1$ for all $x$ in $X$. If $(X, S)$ is a transformation semigroup (briefly a $\tau$-semigroup), each $s$ in $S$ induces a continuous linear transformation $T_{s}$ in $m(X)$ defined by: $\left(T_{s} f\right)(x)=f(s x)$. A mean $\mu$ on $m(X)$ will be called $S$-invariant if $\mu\left(T_{s} f\right)=\mu(f)$ for all $s$ in $S$ and all $f$ in $m(X)$. A $\tau$-semigroup $(X, S)$ will be called $S$-amenable (or we say that $m(X)$ has an $S$-invariant mean) in case there exists an $S$-invariant mean on $m(X)$.

The $l$ - and $r$-semigroups have been studied for amenability extensively in recent years; for example see [1] or [7] for an introduction to the subject, and [3] for a survey with a rather complete bibliography. In these cases means are called left or right invariant, and semigroups having a left [right] invariant mean are called left [right] amenable. The $l$ - and $r$-semigroups are very special, and certain results in the theory of amenable semigroups hold because of their special properties. For example, some results are derived from the fact that if $S$ is an abstract semigroup, then there is associated with $X=S$ as the underlying set two $\tau$-semigroups (left and right multiplication), and some of the theory of amenable semigroups is based on the interplay between these $\tau$-semigroups. In this paper we study general $\tau$-semigroups for amenability. We offer a survey for

1 Both authors supported by the Office of Naval Research. 
convenient reference of analogues of some of the important results known for the $l$ - and $r$-semigroups, and we point out certain contrasts in the theories. Most of our results are not conceptually new; our main goals are to gain generality and to obtain greater insight in the special $l$ - and $r$-semigroup cases.

\section{Amenability of $\tau$-semigroups}

We begin by studying the connection of amenability of general $\tau$-semigroups with that of the $l$ - and $r$-semigroups. The following lemma is used in the proofs of several of the theorems.

LEMMA 1. If $X$ and $Y$ are sets and $A$ is a (continuous) linear, monotonic and normalized mapping of $m(Y)$ into $m(X)$, then the adjoint $A^{*}$ preserves means.

Proof. If $f \in m(Y)$ and $f(y) \geqq 0$ for all $y$ in $Y$, then $A f(x) \geqq 0$ for all $x$ in $X$; hence if $\mu$ is a mean on $m(X)$, then $A^{*} \mu(f)=\mu(A f) \geqq 0$. Since $A 1_{Y}=1_{X}$, $A^{*} \mu\left(1_{Y}\right)=1$.

THEOREM 1. Let $(X, S)$ be a $\tau$-semigroup. If $S$, considered as an abstract semigroup, has a left invariant mean, then $m(X)$ has an $S$-invariant mean.

Proof. Denote the left translation operator in $m(S)$ corresponding to $s$ in $S$ by $l_{s}$. Fix $x$ in $X$ and define a mapping $A\left(=A_{x}\right)$ on $m(X)$ into $m(S)$ by:

$$
A f(s)=f(s x) \text { for } s \text { in } S .
$$

Then $A$ is continuous, linear, monotonic and preserves the constant functions; by lemma 1 the adjoint $A^{*}$ preserves means. Let $\mu$ be a left invariant mean on $m(S)$ and put $v=A^{*} \mu$; it remains to show that $v$ is $S$-invariant. Let $s \in S$ and $f \in m(X)$; then $A\left(T_{s} f\right)=l_{s}(A f)$, for if $t \in S$, then

$$
\left[l_{s}(A f)\right](t)=A f(s t)=f((s t) x)=f(s(t x))=T_{s} f(t x)=\left[A\left(T_{s} f\right)\right](t) .
$$

Hence

$$
A^{*} \mu\left(T_{s} f\right)=\mu\left(A\left(T_{s} f\right)\right)=\mu\left(l_{s}(A f)\right)=\mu(A f)=A^{*} \mu(f) .
$$

The converse of theorem 1 fails, and the conclusion of theorem 1 fails if 'left' is replaced by 'right' in the hypothesis, as the following simple examples show. Take $X=\{a, b, c\}$, and define five transformations, $e, s, t, u, v$, according to the following table:

\begin{tabular}{c|c|c|c|c|c} 
& $e$ & $s$ & $t$ & $u$ & $v$ \\
\hline$a$ & $a$ & $a$ & $a$ & $a$ & $b$ \\
\hline$b$ & $b$ & $b$ & $c$ & $a$ & $b$ \\
\hline$c$ & $c$ & $b$ & $c$ & $a$ & $b$
\end{tabular}


the notation meaning, for example, that $t(b)=c$. Let $S=\{e, s, t\}, S^{\prime}=\{e, u, v\}$. Then $(X, S)$ and $\left(X, S^{\prime}\right)$ are $\tau$-semigroups, $m(X)$ has an $S$-invariant mean, $m(X)$ has no $S^{\prime}$-invariant mean, but as abstract semigroups $S$ and $S^{\prime}$ are isomorphic and have right invariant means but no left invariant means (these assertions will be established in section 3 ).

The examples given above also show that amenability of $\tau$-semigroups is not a semigroup property in the sense of being invariant under isomorphisms of the abstract semigroups involved. Thus a stronger notation of isomorphism is needed.

Definition 1. Let $(X, S)$ and $(Y, T)$ be $\tau$-semigroups. A homomorphism of $(X, S)$ into $(Y, T)$ is a pair of functions $(\phi, \eta), \phi: X \rightarrow Y, \eta: S \rightarrow T$, such that $\phi(s x)=\eta(s) \phi(x)$ for all $s$ in $S, x$ in $X$.

Call $(Y, T)$ a homomorphic image of $(X, S)$ if there exists a homomorphism $(\phi, \eta)$ such that $\phi$ and $\eta$ are both onto.

ReMARK 1. If $(Y, T)$ is a homomorphic image of $(X, S)$, then $\eta$ is a semigroup homomorphism. For let $s_{1} \in S, s_{2} \in S, y \in Y$ and choose $x$ in $X$ such that $\phi(x)=y$; then

$$
\begin{aligned}
\left(\eta\left(s_{1}\right) \eta\left(s_{2}\right)\right)(y) & =\eta\left(s_{1}\right)\left(\eta\left(s_{2}\right)(y)\right)=\eta\left(s_{1}\right)\left(\phi\left(s_{2} x\right)\right)=\phi\left(s_{1} s_{2} x\right)= \\
& =\eta\left(s_{1} s_{2}\right) \phi(x)=\eta\left(s_{1} s_{2}\right)(y) .
\end{aligned}
$$

RemarK 2. The notion of homomorphism defined here has all the desirable features of homomorphisms in general. Namely, if $(Y, T)$ is a homomorphic image of $(X, S)$ under $(\phi, \eta)$, then $X$ and $S$ are both partitioned into mutually disjoint equivalence classes under the relations $x_{1} \sim x_{2}$ iff $\phi\left(x_{1}\right)=\phi\left(x_{2}\right)$ and $s_{1} \approx s_{2}$ iff $\eta\left(s_{1}\right)=\eta\left(s_{2}\right)$. The equivalence classes in $S$ can be regarded as acting on those in $X$; i.e., if $A$ is in a class in $S$ and $E$ a class in $X$, choose $s$ in $A, x$ in $E$ and let $A(E)=F$, where $F$ is the class of $s x$. The action of $A$ at $E$ is well defined since $x_{1} \sim x_{2}$ and $s_{1} \approx s_{2}$ imply $s_{1} x_{1} \sim s_{2} x_{2}$ :

$$
\phi\left(s_{1} x_{1}\right)=\eta\left(s_{1}\right) \phi\left(x_{1}\right)=\eta\left(s_{2}\right) \phi\left(x_{2}\right)=\phi\left(s_{2} x_{2}\right) .
$$

Denote the quotients $X / \sim$ by $X^{\prime}$ and $S / \approx$ by $S^{\prime}$. Then $S^{\prime}$ is a set of transformations on $X^{\prime}$, and it is easy to see that $\left(X^{\prime}, S^{\prime}\right)$ is in fact a $\tau$-semigroup. Further, the fundamental theorem on homomorphisms remains valid in this context. That is $\left(X^{\prime}, S^{\prime}\right)$ is isomorphic to $(Y, T)$ under the natural mappings $\psi(E)=\phi(x)$, where $x \in E$, and $\xi(A)=\eta(s)$, where $s \in A$. We indicate only one part of the proof: given $A$ and $E$, choose $s$ in $A, x$ in $E$; then $A E$ is the class containing $s x$, so that $\psi(A E)=\phi(s x)=\eta(s) \phi(x)=\xi(A) \psi(E)$, and this is the basic relationship in definition 1 .

THEOREM 2. Let $(X, S)$ and $\left(X^{\prime}, S^{\prime}\right)$ be $\tau$-semigroups and suppose that $\left(X^{\prime}, S^{\prime}\right)$ is a homomorphic image of $(X, S)$. Then $m\left(X^{\prime}\right)$ has an $S^{\prime}$-invariant mean if $m(X)$ has an S-invariant mean. 
Proof. Define a mapping $A: m\left(X^{\prime}\right) \rightarrow m(X)$ by: $A f=f \circ \phi$. Then $A$ is continuous, linear and monotonic, and $A\left(1_{X^{*}}\right)=1_{X}$; hence $A^{*}$ preserves means. Let $\mu$ be an $S$-invariant mean, and denote the translation operator in $m\left(X^{\prime}\right)$ also by $T$. Then for $s^{\prime}$ in $S^{\prime}, f$ in $m\left(X^{\prime}\right)$ and $x$ in $X$ we have

$$
\begin{aligned}
\left(A\left(T_{s^{\prime}} f\right)\right)(x)=\left(T_{s^{\prime}} f\right) \phi(x) & =f\left(s^{\prime}(\phi(x))\right)=f(\eta(s) \phi(x)) \\
& =f(\phi(s x))=A f(s x)=\left(T_{s}(A f)\right)(x),
\end{aligned}
$$

where $s \in S$ and $\eta(s)=s^{\prime}$. Thus $A\left(T_{s^{\prime}} f\right)=T_{s}(A f)$, so that

$$
A^{*} \mu\left(T_{s^{\prime}} f\right)=\mu\left(A\left(T_{s^{\prime}} f\right)\right)=\mu\left(T_{s}(A f)\right)=\mu(A f)=A^{*} \mu(f) .
$$

The next theorem contains another sufficient condition for the existence of $S$-invariant means. It was proved in [1] for the $l$-and $r$-semigroups, but the proof given there cannot be carried over to the general case without significant modification.

THEOREM 3. Let $(X, S)$ be a $\tau$-semigroup. If $Y \subseteq X$ such that $s[Y] \subseteq Y$ for all $s$ in $S$, let $R$ be the set of transformations of $Y$ obtained by restricting the mappings $s$ in $S$ to $Y$, with two mappings identified if they agree on $Y$. If $m(X)$ has an $S$ invariant mean $\mu$ such that $\mu\left(\chi_{Y}\right)>0$, then $Y$ has an R-invariant mean $(\chi$ denotes characteristic function).

Proof. Define $A: m(Y) \rightarrow m(X)$ by:

$$
A f(x)= \begin{cases}f(x) & \text { if } x \in Y \\ 0 & \text { if } x \notin Y .\end{cases}
$$

Then $A$ is continuous, linear and monotonic, and $A\left(1_{Y}\right)=\chi_{Y}$. Hence if we put $v=\left(1 / \mu\left(\chi_{Y}\right)\right) A^{*} \mu$, then $v$ is a mean on $m(Y)$, and it remains to show that $v$ is $R$-invariant. From this point on our proof must be different from that given in [1]. If $t \in R$, choose $s$ in $S$ such that $\left.s\right|_{Y}=t$. Then for $f$ in $m(Y)$ and $x$ in $X$ we have

and

$$
\left(A\left(T_{t} f\right)\right)(x)= \begin{cases}f(t x)=f(s x) & \text { if } x \in Y \\ 0 & \text { if } x \notin Y,\end{cases}
$$

$$
\left(T_{s}(A f)\right)(x)= \begin{cases}f(s x) & \text { if } s x \in Y \\ 0 & \text { if } s x \notin Y .\end{cases}
$$

Hence $A\left(T_{t} f\right)$ and $T_{s}(A f)$ agree except possibly on the set $E_{1}=\tilde{Y} \cap s^{-1}[Y]$. Now for $n$ an integer, $n \geqq 2$, put $E_{n}=s^{-1}\left[E_{n-1}\right]$. By induction, if $n \geqq 2$, then $x \in E_{n}$ iff $s^{n-1} x \in \widetilde{Y}$ and $s^{n} x \in Y$. Hence the sets $E_{n}$ are pairwise disjoint, and

$$
\mu\left(\chi_{E_{n}}\right)=\mu\left(\chi_{s^{-1}\left[E_{n-1}\right]}\right)=\mu\left(T_{s} \chi_{E_{n-1}}\right)=\mu\left(\chi_{E_{n-1}}\right) .
$$

It follows that $\mu\left(\chi_{E_{1}}\right)=0$. By the Riesz representation theorem, there exists a unique regular Borel measure $\bar{\mu}$ of total mass 1 on the Stone-Čech compactifica- 
tion of the discrete space $X$ such that for each $g$ in $m(X)$ we have $\mu(g)=\int_{\beta X} \hat{g} d \bar{\mu}$, where $\hat{g}$ is the unique continuous extension of $g$ to $\beta X$. Then

$$
\begin{aligned}
\mu\left(A\left(T_{t} f\right)\right)-\mu\left(T_{s}(A f)\right) & =\mu\left(A\left(T_{t} f\right)-T_{s}(A f)\right) \\
& =\int_{\beta X}\left(A\left(T_{t} f\right)-T_{s}(A f)\right) \hat{\mathrm{d}} \bar{\mu} \\
& =\int_{\bar{E}_{1}}\left(A\left(T_{t} f\right)-T_{s}(A f)\right) \hat{\mathrm{d}} \bar{\mu},
\end{aligned}
$$

where $\bar{E}_{1}$ denotes the ( $\left.w^{*}\right)$ closure of $E_{1}$ in $\beta X$, since the integrand vanishes on $\beta X \sim \bar{E}_{1}$. Since $\bar{\mu}\left(\bar{E}_{1}\right)=\mu\left(\chi_{E_{1}}\right)=0$, it follows that $\mu\left(A\left(T_{t} f\right)\right)=\mu\left(T_{s}(A f)\right)=$ $\mu(A f)$, and hence $v$ is $R$-invariant.

Before turning to characterizations of amenable $\tau$-semigroups we make two further observations. First, given $\tau$-semigroups $(X, S)$ and $(Y, T)$, the pair $(X \times Y, S \times T)$ becomes a $\tau$-semigroup with the action of $(s, t)$ at $(x, y)$ defined to be $(s x, t y)$. If $m(X)$ has an $S$-invariant mean and $m(Y)$ has a $T$-invariant mean, then $m(X \times Y)$ has an $S \times T$-invariant mean, defined just as the product of two measures.

The second observation arises from an attempt to extend the notion of ideals to $\tau$-semigroups. The natural generalization of the concept of a left ideal to $(X, S)$ is an invariant set, i.e. a set $Y \subseteq X$ for which $s[Y] \subseteq Y$ for all $s$ in $S$. Here is another point of contrast between the general and the $l$ - and $r$-semigroups. For it is true that if $Y$ is an invariant set, if $T$ consists of the restrictions of the mappings in $S$ to $Y$ and if $m(X)$ has a $T$-invariant mean, then $m(X)$ has an $S$-invariant mean; a proof can be constructed along the lines of the proofs given for theorems 1-3. It was proved in [11] that the converse is valid for $l$-semigroups. However, the converse fails in general, as the example $(X, S)$ given after theorem 1 shows. In that example $m(X)$ has an $S$-invariant mean, the set $Y=\{b, c\}$ is invariant, but $m(Y)$ does not have a $T$-invariant mean for $T$, the set of restrictions of elements of $S$ to $Y$.

\section{Characterizations of amenable $\tau$-semigroups}

For $x$ in $X$ denote by $q x$ the evaluation functional defined on $m(X)$ by: $q x(f)=f(x)$. Then $q x$ is a mean on $m(X)$ for all $x$ in $X$. In fact, the set of all means on $m(X)$ is $w^{*}$-compact and convex, and each $q x$ is an extreme point of this set; it is a consequence of the Krein-Mil'man theorem that the set of all means coincides with $\overline{c o} q[s]$. Further the ( $w^{*}$-) closure of $q[s]$ coincides with $\beta X$, the Stone-Cech compactification of the discrete space $X$.

We can now establish the assertions concerning the examples given after theorem 1. A finite abstract semigroup has a left invariant mean if and only if each pair of right ideals has a nonempty intersection (Rosen [10]); in $S$, 
$s S \cap t S=\emptyset$. The points $s$ and $t$ are left zeros of $S$ and hence $q s$ and $q t$ are right invariant means. For $(X, S) q a$ is an $S$-invariant mean since $a$ is fixed under each element of $S$. Suppose $m(X)$ has an $S^{\prime}$-invariant mean $\mu$. Then, $\mu$ must be of the form

$$
\mu=\alpha_{1} q a+\alpha_{2} q b+\alpha_{3} q c, \quad \alpha_{1}+\alpha_{2}+\alpha_{3}=1 .
$$

Choose $f \in m(X)$ such that $f(a)=0$ and $f(b)=1$. Then, $\mu\left(T_{u} f\right) \neq \mu\left(T_{v} f\right)$ since $T_{u} f=0$ and $T_{v} f=1$; this is a contradiction.

In the case of the $l$ - and $r$-semigroups, the set of all means becomes a semigroup under the Arens multiplication, and this semigroup has a number of interesting properties (see [11] for details). For example, if $X(=S)$ has a left invariant mean, then the smallest closed two-sided ideal in the semigroup of means consists of all the left invariant means. In the general case the Arens multiplication is not available, but it is possible to define a mapping, which we denote by juxtaposition, of $m(S)^{*} \times m(X)^{*}$ into $m(X)^{*}$ by: $\mu v(f)=\xi\left(\phi_{v} f\right)$, where $\phi_{v} f$ is the element in $m(S)$ whose action at $s$ in $S$ is: $\phi_{v} f(s)=v\left(T_{s} f\right)$. The basic properties of the mapping defined here are given in the following lemma which we state without proof; they are easy to check.

LEMMA 2. The operation defined above has the properties:

(i) $\|\mu v\| \leqq\|\mu\| \cdot\|v\|$;

(ii) for a fixed $\mu$ in $m(S)^{*}\left[v\right.$ in $\left.m(X)^{*}\right]$ the mapping $v \rightarrow \mu v[\mu \rightarrow \mu v]$ is a continuous linear mapping of $m(X)^{*} \rightarrow m(X)^{*}\left[m(S)^{*} \rightarrow m(X)^{*}\right]$;

(iii) if $w^{*}-\lim _{n} \mu_{n}=\mu$ in $m(X)^{*}$, then $w^{*}-\lim _{n} \mu_{n} v=\mu v$ in $m(X)^{*}$ for each $v$ in $m(X)^{*}$;

(iv) if $w^{*}-\lim _{n} v_{n}=v$ in $m(X)^{*}$ and $\theta$ is a finite mean (i.e. the carrier of $\theta$ is finite), then $w^{*}-\lim _{n} \theta v_{n}=\theta v$ in $m(X)^{*}$;

(v) $q s q x=q s x$ for each $s$ in $S, x$ in $X$.

Call a net $\left\{\theta_{n}\right\}$ of finite means $\left(w^{*}\right)$ convergent [strongly convergent] to $S$-invariance if $w^{*}-\lim _{n}\left(q s \theta_{n}-\theta_{n}\right)=0\left[\lim _{n}\left\|q s \theta_{n}-\theta_{n}\right\|=0\right]$ for each $s$ in $S$. If $\mu$ is an $S$-invariant mean and $\left\{\theta_{n}\right\}$ is a net of finite means such that $w^{*}-\lim \theta_{n}=\mu$, then $\left\{\theta_{n}\right\}$ converges to $S$-invariance. Conversely, if $\left\{\theta_{n}\right\}$ converges to $S$-invariance, then any $w^{*}$-cluster point is an $S$-invariant mean. Hence a $\tau$-semigroup has an $S$-invariant mean if and only if there is a net of finite means converging to $S$-invariance.

The following theorem was first proved by Day in [1]. Namioka [9] gave an elegant proof of Day's theorem, and Namioka's proof can be carried over to $\tau$-semigroups with only the slight modification of replacing $\left(l_{1}(S)\right)^{S}$ by $\left(l_{1}(X)\right)^{S}$ (see [9]).

Theorem 4 (Day). Let $(X, S)$ be a $\tau$-semigroup. Then $X$ has an $S$-invariant mean if and only if there exists a net $\left\{\theta_{n}\right\}$ of finite means on $X$ such that

$$
\lim \left\|q s \theta_{n}-\theta_{n}\right\|=0 \text {. }
$$


In [9], Namioka also gave an elegant proof of the Følner-Frey theorem on amenable semigroups. Only a slight change is required to adapt the computations to $\tau$-semigroups. Specifically, in place of the convolution in $l_{1}(S)$ define a mapping in $l_{1}(X)$ as follows: for each $s$ in $S$ and each $\theta=\sum_{i=1}^{n} \lambda_{i} \delta x_{i}$ in $l_{1}(X)\left(\lambda_{i} \geqq 0\right.$, $\sum_{i=1}^{n} \lambda_{i}=1$ and $\delta$ is the Kronecker embedding of $X$ into $\left.l_{1}(X)\right)$ put $s \cdot \theta=$ $\sum_{i=1}^{n} \lambda_{i} \delta s x_{i}$. Under this analogue of convolution in $l_{1}(X)$ all of Namioka's computations are valid, and the following characterization of amenable $\tau$-semigroups is obtained.

THEOREM 6 (Følner-Frey). Let $(X, S)$ be a t-semigroup. Then $m(X)$ has an $S$-invariant mean if and only if given any finite set $F$ in $S$ and $\varepsilon>0$, there exists $a$ finite set $A$ in $X$ such that $|s[A] \sim A|<\varepsilon|A|$ for each $s$ in $F$.

In [2], Day established a characterization of abstract semigroups with left (or right) invariant means in terms of the Markov-Kakutani fixed point property. This theorem also has an analogue in $\tau$-semigroups under the stronger notion of homomorphism.

TheOREM 7 (Markov-Kakutani-Day). Let $(X, S)$ be a $\tau$-semigroup. A necessary and sufficient condition that $m(X)$ have an $S$-invariant mean is that whenever $K$ is a compact convex set in a locally convex linear topological space $E$ and $S^{\prime}$ is a semigroup (under composition) of continuous affine mappings of $K$ such that $\left(K, S^{\prime}\right)$ is a homomorphic image of $(X, S)$, there is a point $y$ in $K$ such that ty $=y$ for all $t$ in $S^{\prime}$.

Proof. Sufficiency. The pair $(\phi, \eta)$, where $\phi=q$, the evaluation mapping, and $\eta(s)=T_{s}^{*}$, is a homomorphism (each $T_{s}^{*}$ is restricted to the set of means on $m(X)$, which is equipped with the $w^{*}$-topology); the common fixed point of the mappings $T_{s}^{*}$ is an $S$-invariant mean on $m(X)$.

Necessity. Denote the canonical embedding of $E$ into $E^{* *}$ by $Q$; then $Q$ is an affine homeomorphism of $K$ into $Q[K]$. Let $(\phi, \eta)$ be the homeomorphism of $(X, S)$ onto $\left(K, S^{\prime}\right)$, and define $A: E^{*} \rightarrow m(X)$ by: $A f=f \circ \phi$. Then $A^{*} q x=$ $Q \phi(x)$, so that $Q^{-1} A^{*} q=\phi$. Moreover $Q^{-1} A^{*}$ is a continuous affine mapping of the set of means on $m(X)$, and if $\mu$ is an $S$-invariant mean on $m(X)$, then $Q^{-1} A^{*} \mu$ is a common fixed point for $S^{\prime}$. For let $\left\{\theta_{n}\right\}$ be a net of finite means converging $\left(w^{*}\right)$ to $\mu$. Each $\theta_{n}$ is of the form

$$
\theta_{n}=\sum_{i=1}^{N(n)} \lambda_{i}^{n} q x_{i}^{n}
$$

with each $\lambda_{i}^{n} \geqq 0$ and $\sum_{i=1}^{N(n)} \lambda_{i}^{n}=1$ for each $n$. Then

$$
Q^{-1} A^{*} \theta_{n}=\sum_{i=1}^{N(n)} \lambda_{i}^{n} \phi\left(x_{i}^{n}\right)
$$

and $\theta^{-1} A^{*} \theta_{n} \rightarrow Q^{-1} A^{*} \mu$. Moreover, if $t \in S^{\prime}$, then $t\left(Q^{-1} A^{*} \theta_{n}\right) \rightarrow t\left(Q^{-1} A^{*} \mu\right)$, and 


$$
t\left(Q^{-1} A^{*} \theta_{n}\right)=\sum_{i=1}^{N(n)} \lambda_{i}^{n} t \phi\left(x_{i}^{n}\right)=\sum_{i=1}^{N(n)} \lambda_{i}^{n} \phi\left(s x_{i}^{n}\right)
$$

where $s \in S$ and $\eta(s)=t$. Put

$$
\psi_{n}=\sum_{i=1}^{N(n)} \lambda_{i}^{n} q s x_{i}^{n}
$$

then $t\left(Q^{-1} A^{*} \theta_{n}\right)=Q^{-1} A^{*} \psi_{n}$, and $\psi_{n} \rightarrow \mu$ since $\mu$ is $S$-invariant. Hence $t\left(Q^{-1} A^{*} \theta_{n}\right) \rightarrow Q^{-1} A^{*} \mu$, and therefore $t\left(Q^{-1} A^{*} \mu\right)=Q^{-1} A^{*} \mu$.

REMARK 3. Theorem 6 includes the form of the Markov-Kakutani theorem given by Day in [2] as a special case. In this case $S$ is an abstract semigroup, and we take $X=S$ as the underlying set and define the action of $s$ in $S$ at $x$ in $X$ by $s(x)=s x$. Let $h$ be a homomorphism of $S$ onto $S^{\prime}$. Choose $y$ from $K$ and define a homomorphism $(\phi, \eta)$ of $(X, S)$ into $\left(K, S^{\prime}\right)$ by: $\phi(x)=(h(x))(y)$ and $\eta(s)=h(s)$. The pair $(\phi, \eta)$ is in fact a homomorphism since

$$
\begin{aligned}
\phi(s x) & =(h(s x))(y)=(h(s) h(x))(y)=h(s)((h(x))(y)) \\
& =h(s) \phi(x)=\eta(x) \phi(s) .
\end{aligned}
$$

The concept of extremely amenable semigroups was introduced by Mitchell in [8] and studied extensively by Granirer in [4], [5], [6]. Using an argument analogous to that given in theorem 6 , we obtain the corresponding result for extremely amenable $\tau$-semigroups, i.e. $\tau$-semigroups $(X, S)$ where $m(X)$ has an $S$-invariant mean which lies in $\beta X$.

THEOREM 8. Let $(X, S)$ be a $\tau$-semigroup. A necessary and sufficient condition that $m(X)$ have an $S$-invariant mean $\mu$ in $\beta X$ is that whenever $Y$ is a compact Hausdorff space and T is a semigroup of continuous mappings on $Y$ such that $(Y, T)$ is a homomorphic image of $(X, S)$, there is a point $y$ in $Y$ such that ty $=y$ for all $t$ in $T$.

Remark 4. Another striking difference between the theories of amenability of general $\tau$-semigroups and of the $l$ - and $r$-semigroups appears in connection with multiplicative invariant means. Granirer [4] characterized extremely left amenable semigroups by the property: given $s, t$ in $S$, there exists $u$ in $S$ such that $s u=t u=u$. Thus a nontrivial semigroup with right cancellation cannot be extremely left amenable. The situation is different for general $\tau$-semigroups. For take $X=\{a, b, c\}, s$ the identity on $X$ and $t$ defined by $t(a)=a, t(b)=c$, $t(c)=b$. Then $(X,\{s, t\})$ is a $\tau$-semigroup, $S=\{s, t\}$ forms a group, and the integral with respect to unit mass at $a$ is $S$-invariant.

Remark 5. Granirer also showed in [4] that the existence of a multiplicative left invariant mean is equivalent to the existence of a net of point measures converging strongly to left invariance (in the sense of theorem 4 above). In simple cases such as finite $\tau$-semigroups an $S$-invariant mean in $\beta X$ must be the integral 
with respect to unit mass concentrated at a common fixed point of all $s$ in $S$. An interesting problem is to determine whether either of Granirer's characterizations hold for $\tau$-semigroups. In this connection we note that for a $\tau$-semigroup $(X, S)$ if $S$, when considered as an abstract semigroup, has a multiplicative left invariant mean, then $m(X)$ has an $S$-invariant mean in $\beta X$. This follows from the proof of theorem 1 together with the observation that for each $s$ in $S A^{*} q s=q(s x)$ (see lemma 2 and theorem 1 for notation).

\section{References}

[1] M. M. Day, 'Amenable semigroups', Ill. J. Math. 1 (1957), 509-544.

[2] M. M. Day, 'Fixed point theorems for compact convex sets', Ill. J. Math. 5 (1961), 585-590.

[3] M. M. Day, 'Semigroups and amenability', to appear in the Proceedings of a Symposium on Semigroups at Wayne State University, June 1968, Academic Press.

[4] E. Granirer, 'Extremely amenable semigroups I', Math. Scand. 17 (1965), 177-197.

[5] E. Granirer, 'Extremely amenable semigroups II', Math. Scand. 20 (1967), 93-113.

[6] E. Granirer, 'Extremely amenable semigroups III', to appear.

[7] E. Hewitt and K. Ross, Abstract Harmonic Analysis, Vol. 1. Springer-Verlag, BerlinGöttingen-Heidelberg, 1963.

[8] T. Mitchell, 'Fixed points and multiplicative left invariant means', Trans. Amer. Math. Soc. 122 (1966), 195-202.

[9] I. Namioka, 'Følner's condition for amenable semigroups', Math. Scand. 15 (1964), 18-28.

[10] W. G. Rosen, 'On invariant means over compact semigroups', Proc. Amer. Math. Soc. 7 (1956), 1076-1082.

[11] C. Wilde and K. Witz, 'Invariant means and the Stone-Cech compactification', Pac. $J$. Math. 21 (1967), 577-586.

Naval Postgraduate School

Monterey 